\title{
Civil Structure Condition Assessment by FE Model
}

\section{Updating: Methodology and Case Studies}

\section{James M W Brownjohn}

Associate Professor

School of Civil and Structural Engineering, Nanyang Technological University, Nanyang Avenue, Singapore 639798

\section{Pin-Qi Xia}

PhD Student

School of Civil and Structural Engineering, Nanyang Technological University, Nanyang Avenue, Singapore 639798

\section{Hong Hao}

Associate Professor

School of Civil and Structural Engineering, Nanyang Technological University, Nanyang Avenue, Singapore 639798

\section{Yong Xia}

PhD Student

School of Civil and Structural Engineering, Nanyang Technological University, Nanyang Avenue, Singapore 639798

Author responsible for correspondence:

Professor James M W Brownjohn

N1-1a-11

Division of Structures and Mechanics

School of Civil and Structural Engineering

Nanyang Technological University

50 Nanyang Avenue

Singapore 639798

Telephone: $+65-7904773$

Fax: +65-7910676

Email: cjames@ntu.edu.sg 


\begin{abstract}
Development of methodology for accurate and reliable condition assessment of civil structures has become increasingly important. In particular, the finite element (FE) model updating method has been successfully used for condition assessment of bridges. However, the success of applications of the method depends on the analytical conceptualization of complex bridge structures, a well-designed and controlled modal test and an integration of analytical and experimental arts. This paper describes the sensitivity analysis based FE model updating method and its application to structure condition assessment with particular reference to bridges, including specific considerations for FE modeling for updating and the model updating procedure for successful condition assessment. Finally, the accuracy analysis of damage assessment by model updating was investigated through a case study.
\end{abstract}

Keywords: Condition assessment; civil structure; finite element model updating; damage; sensitivity analysis; modal analysis.

\title{
1. Introduction
}

It has been reported [1] that about 125,000 of the 585,000 bridges in the USA are deemed deficient. Condition assessment of bridges is largely based on visual observations and described by subjective indices which do not permit accurate evaluation of bridge dynamics, serviceability and safety. Because reliable assessment depends on quantitative rather than qualitative information, there is an urgent need and essential motivation to develop methodologies for objective and accurate condition assessment and reliability evaluation. 
A significant amount of research has been performed on the condition assessment of existing bridges and relevant research has accelerated in recent years. Aktan et al. [1,2,3] described an integrated experimental and analytical methodology for structural identification and field testing aimed at condition assessment of bridges, through consideration of defects, deterioration, damage, bridge state and performance. Saraf [4] used nondestructive load testing methods to evaluate three existing reinforced concrete bridges. Wahab and Roeck [5] investigated the damage detection in bridges using curvatures of mode shapes. These investigations were based on the field testing and numerical analysis. The condition of these bridges was still difficult to assess quantitatively. The difficulty is that the deterioration and damage in the structure are difficult to describe mathematically.

The finite element (FE) model updating method [6,7] has emerged in the 1990s as a subject of great importance for mechanical and aerospace structures. However, this updating technology can be difficult to apply as an engineering tool for civil engineering structures, because of the difficulties in prototype testing and experimental data analysis resulting from the nature, size, location and usage of these structures. Only recently, the civil engineering community has begun to adopt this technology. Cantieni [8] investigated model updating of a concrete arch bridge while Pavic et al. [9] and Reynolds et al. [10] applied the technique to footbridges and concrete floors. Research on different applications of the model updating method to damage assessment of a model bridge for wind tunnel testing, dynamic assessment of a cable-stayed bridge, residual stiffness assessment of a failed reinforced concrete (RC) slab, and load-carrying capacity of a 
damaged $\mathrm{RC}$ beam bridge structure has been investigated by Brownjohn and Xia $[11,12,13,14]$.

Since the FE model updating method integrates several disciplines, the success of its application to bridge condition assessment depends mainly on the interdisciplinary experience and skill of the analyst. Meanwhile, it is expected that its application to civil engineering structures, especially to bridge structures will become popular. Hence, it is extremely valuable to summarize the experience and methodology. Based on this motivation, this paper describes the sensitivity analysis based FE model updating method and its application to bridge condition assessment, including the specific techniques in the FE modeling for model updating and the actual model updating procedure. In order to verify the structural condition assessment by the model updating, the accuracy analysis of damage assessment by the method was investigated through a case study of a damaged steel portal frame.

\section{Sensitivity based Updating Method}

The FE model updating method was developed through combining the FE analysis (FEA) with the experimental modal analysis (EMA). The aim was to correct the geometrical and/or physical parameters and/or boundary conditions of the initial FE model through a model tuning procedure based on the experimental results such as measured frequencies and mode shapes etc., and further to reproduce the dynamic performances of a structure with greatest accuracy compared with the experimental results. The FEA and EMA integrated analysis can remedy the deficiencies of using merely numerical analysis and obtain great improvement of numerical results. 
In terms of the principle of FE model updating, the system matrices (mass, stiffness and possibly damping matrices) that completely describe the FEA are modified or updated with respect to the reference data which are generally the experimental modal parameters such as measured frequencies and mode shapes. Based on the modification of system matrices, model updating procedures can be classified as being iterative (local methods) or as one-step procedures (global methods). Local methods are based on corrections applied to local physical parameters of the FE model, and therefore are physically meaningful. Global methods directly reconstruct the updated global mass and stiffness matrices from the reference data, so lack the advantages of local methods. The effective and most popular local methods for model updating are generally based on the sensitivity analysis. In the formulation of parameter modification in FE models, the 'true' (or experimental) responses, such as frequencies or mode shapes, are expressed as functions of analytical responses, structural parameters and a sensitivity coefficient matrix. This is done in terms of a first order Taylor series as follows:

$$
\left\{R_{e}\right\}=\left\{R_{a}\right\}+[S]\left(\left\{P_{u}\right\}-\left\{P_{o}\right\}\right)
$$

or

$$
\{\Delta R\}=[S]\{\Delta P\}
$$

where $\{\Delta R\}=\left\{R_{e}\right\}-\left\{R_{a}\right\},\left\{R_{e}\right\}$ and $\left\{R_{a}\right\}$ are vectors of experimental and analytical response values, respectively. $\{\Delta P\}=\left\{P_{u}\right\}-\left\{P_{o}\right\},\left\{P_{u}\right\}$ and $\left\{P_{o}\right\}$ are vectors of updated and current iterative parameter values, respectively. $[S]$ is sensitivity matrix. For all selected responses and parameters, the sensitivity matrix $[S]$ can be calculated as follows: 


$$
[S]_{i j}=\frac{\partial R_{i}}{\partial P_{j}}
$$

where, $R_{i}$ and $P_{j}$ represent a structural response and parameter, respectively. The subscript $i=1 \ldots N$ for $N$ responses and $j=1 \ldots M$ for $M$ parameters. The sensitivity matrix can be computed for all physical element properties (material, geometrical, boundary, etc.) by using direct derivation or perturbation techniques. This depends on whether mass and stiffness are proportional or non-proportional to the property.

Equation (3) calculates the absolute sensitivities. This means that they use the units of the response and parameter values. If sensitivities for different types of parameters are to be compared, then the relative sensitivity matrix $\left[S_{r}\right]$ is used and defined by:

$$
\left[S_{r}\right]_{i j}=\left[\frac{\partial R_{i}}{\partial P_{j}}\right]\left[P_{j}\right]
$$

Furthermore, the relative sensitivities can be normalized with respect to the response value. The resulted sensitivities form the normalized relative sensitivity matrix $\left[S_{n}\right]$ which is defined by:

$$
\left[S_{n}\right]_{i j}=\left[R_{i}\right]^{-1}\left[\frac{\partial R_{i}}{\partial P_{j}}\right]\left[P_{j}\right]
$$

Equation (2) may be determined, over-determined or under-determined depending on whether the number of responses $N$ is equal to, larger than or smaller than the number of parameters $M$, respectively. In any case, the parameter modification $\{\Delta P\}$ in equation (2) can be solved using the pseudo-inverse technique [15]:

$$
\{\Delta P\}=[S]^{+}\{\Delta R\}
$$

where, $[S]^{+}$is the pseudo-inverse matrix of sensitivity matrix $[S]$ and is given by: 


$$
[S]^{+}= \begin{cases}{[S]^{-1}} & \text { for } N=M \\ \left([S]^{T}[S]\right)^{-1}[S]^{T} & \text { for } N>M \\ {[S]^{T}\left([S][S]^{T}\right)^{-1}} & \text { for } N<M\end{cases}
$$

$[S]^{+}$can be computed using the Bayesian estimation or singular value decomposition techniques [15].

Since the Taylor's expansion (1) is truncated after the first term, the neglected higher order terms necessitate several iterations, especially when $\{\Delta R\}$ contains large values. It should be noted that when too large discrepancies exist between the experimental and analytical models, the validity of the Taylor series truncations to first order in equation (1) is undermined and iterative process is prone to divergence. Practical consequence of this is that the starting FE model prior to updating should be relatively close to the experimentally measured behavior.

\section{FE Modeling for Updating}

Finite element analysis has for some decades been a powerful tool for simulating structural behavior, but creating a good FE model is not an easy task. Many different modeling strategies and good practice guidelines are available [16] covering the selection of element types, degrees of freedom, appropriate analysis method, etc. These strategies depend on the skill and experience of the analyst, and on the intended application of the model (e.g. static and dynamic FE analysis require different FE models for the same level of accuracy). However, preparation of an FE model that will be a candidate for updating 
requires some specific considerations of additional factors not normally taken into account in conventional FE model construction.

The objective of FE model updating is modification of the inaccuracies or uncertainties in the structure, which must be expressed as parameters so that these inaccuracies/uncertainties can be assessed quantitatively. Therefore, it is vital to successful condition assessment of a structure whether the uncertainties in the structure can be represented by quantitative parameters as far as possible. If uncertainties in structures are not quantified, then the condition assessment of the structures cannot be done.

When damage and/or deterioration are known to exist in a restricted area of a structure, the damaged zones will not generally be contained in a FE model unless some special facilities are incorporated. Because the damage reduces the structure geometrically and/or physically, specific 'weak' elements [11] can be used to represent quantitatively the damaged zones geometrically and physically in the FE model. Of course, determination of the parameters and locations of 'weak' elements is subjective but not optional because it must rely on the damage condition. As long as these 'weak' elements are updated, their updated values represent the condition of damage zones, e.g. damage extent. Fig. 1 shows a kind of 'weak' FE model of damaged joint. Using this idea, the damage in a model bridge [11], the residual stiffness of a failed reinforced concrete slab [13] and the load-carrying capacity of a damaged reinforced concrete beam structure [14] were identified successfully. Fig. 2 shows the FE model of a bridge deck damaged at midspan where four 'weak' beam elements were incorporated into the FE model. 
A common problem in setting up an FE model is to determine the boundary conditions. A good way to simulate boundary conditions is to use support springs such as shown in Fig. 2. Their elastic stiffness values after updating will approximate the real boundary conditions.

In order to obtain a reliable FE model and guarantee the physical significance of the updated parameters after updating, the FE model for updating requires a level of detail to represent geometric and structural form. An oversimplified FE modeling is unlikely to be successful for updating. When performing dynamic assessment of a curved cable-stayed bridge by model updating [12], the authors adapted two FE models of the bridge as candidates for updating. The first model, adapted for checking static load combinations and shown in Fig. 3, featured a 'spine beam' using conventional 3D beam elements to represent the deck girder $[17,18]$. These beam elements supposedly incorporated all the bending, torsional, and inertial properties of the wing-tip box-type deck of the bridge, whereas low density elements capable of transferring static loads, but not representing inertia properties, were used for the deck. The second model, shown in Fig. 4, detailed fully the geometrical properties of the deck in which the structural components of the wing-tip box-type deck were modeled by shell elements. The updated results applied to the first model were not ideal, with a maximum frequency error of $15 \%$. To achieve this level of agreement, six selected parameters changed by $100 \%$, losing their physical relevance. By condensing deck properties into a spine beam model, the original geometrical properties of the wing-tip box-type deck were lost. The updated results for the second model were more successful. All seven frequency differences between 
updated and measured were less than $10 \%$. The maximum changes of five selected parameters were by about $30 \%$.

\section{Model Updating Procedure}

The model updating procedure includes generally three aspects $[11,12]$ :

(1) selection of 'responses' as reference data which are normally the measured data, such as measured frequencies and mode shapes;

(2) selection of parameters to update, to which changes the selected responses should be sufficiently sensitive and must be uncertain; and

(3) model tuning which is an iterative process to modify the selected parameters based on the selected reference data.

Based on these general principles, an updating procedure can be conducted and its more detailed steps will be outlined, as follows.

\section{Selection of Updating Parameters}

In the three aspects of the model updating as described previously, the selection of the parameters to update is crucial because the FE model of the real structure is affected by updating the selected parameters. The important issues are, first, how many parameters should be selected, and, second, which parameters from the many candidates are preferred. Physically, the selected updating parameters must be uncertain in the model. Otherwise, the blindly updated structural components may lose their originally certain properties and produce meaningless results in the updated FE analysis. Mathematically, if the estimation of too many parameters is attempted, then the problem 
may appear ill-conditioned or underdetermined because the observations are limited in vibration testing. To have a well-conditioned updating problem, and bearing in mind the limitation of the measurements, it is necessary to select those updating parameters that will be most effective in producing a genuine improvement in modeling of the structure. Therefore, the number of updating parameters should be kept small, and such parameters should be chosen with the aims of correcting recognized uncertainty in the model and ensuring that the data should be sensitive to them. One good way to assess this is to conduct a sensitivity analysis that computes the sensitivity coefficient defined by equation (3). The sensitivity analysis can be done using optimized proprietary software, such as FEMtools [19] or may be developed around existing FE codes using standard programming tools. When sensitivity analysis is used to help selection of parameters for model tuning, one should start with all possible parameters, identify sensitive and insensitive areas, and then eliminate ineffective (low sensitivity) parameters. Selection of effective parameters can also avoid the ill-conditioning of the sensitivity matrix $[S]$ in equation (2). If some responses are very sensitive to the selected parameters, and others are very insensitive to the same parameters, then ill-conditioning is likely to occur.

\section{Manual Tuning}

As previously mentioned, when doing the model tuning, it is very important to determine a suitable initial value of a selected parameter to provide a reasonable starting point. If the initial value is too different from its true value, and large discrepancies exist between the experimental and analytical models, then $\{\Delta R\}$ in equation (2) may contain large values, which will result in the iterative process diverging. Usually, it is required to 
carry out manual tuning by engineering judgement or relevant preliminarily estimation for obtaining a reasonable approximation before model tuning. This case may happen generally for uncertain parameters from boundary conditions, joints, welding parts, damage or deterioration. The manual tuning procedure, which may also include the addition or subtraction of complete elements or sets of elements is termed 'macroupdating' [20].

\section{Bounds of Updating Parameters}

In order to guarantee the physical significance of updated parameters and avoid physically impossible updated parameter values, the lower and upper bounds for the parameter values should be applied. If a parameter value reaches its allowable extreme during iterative model tuning, then the parameter becomes ineffective during the rest of the procedure. It is possible that convergence cannot be obtained to a satisfactory degree when parameter bounds are defined. A trade-off between physically acceptable parameter values and the convergence level is then required.

\section{Model Evaluation}

After finishing the model tuning, the updated FE model needs to be evaluated so as to determine whether the model updating procedure is successful or not. One way is to use the modal assurance criterion (MAC) [21] to check correlation of mode shapes. It is defined as follows:

$$
\operatorname{MAC}\left(\phi_{a}, \phi_{e}\right)=\frac{\left|\phi_{a}^{T} \phi_{e}\right|^{2}}{\left(\phi_{a}^{T} \phi_{a}\right)\left(\phi_{e}^{T} \phi_{e}\right)}
$$


where, $\phi_{a}$ and $\phi_{e}$ are the analytical and experimental mode shape vectors, respectively.

Given a set of experimental modes and a set of predicted modes, a matrix of $M A C$ values can be computed. The mode shapes with a $M A C$ value equal to $100 \%$ represent a perfect correlation (i.e. linear dependence), whereas modes which are completely orthogonal (i.e. linearly independent) have $0 \% M A C$ value. Generally, it is accepted that a value in excess of $90 \%$ should be attained for correlated modes. For the frequencies, the differences between the analytical and experimental values can be computed. If the $M A C$ values between updated and measured models are high and frequency differences between updated and measured are low, then the model updating is deemed to be successful.

An alternative way to check the reliability of the updated model is to rerun the updated numerical model with parameters perturbed about values of the updated parameters, taking the frequencies and mode shapes as reference data, and perform an updating procedure for the perturbed model [12]. If the perturbed model converges very fast to the updated model with low frequency errors and high $M A C$ values, then the model updating is verified.

\section{Accuracy Analysis}

What is the accuracy of the bridge condition assessment by the method? To answer this question in a controlled laboratory-based study, a damaged steel portal frame was used to compare updated values of the geometrical parameters at damaged zones with their known real values. The frame consisted of three steel strips fixed at two ends and is shown in Fig. 5. Four positions in the frame were cut by saw to simulate the damage. The 
depths of four cuts were all about $80 \%$ of the strip width, therefor producing serious damage in the frame.

The experimental modal analysis was performed on the frame before and after the cut [22]. The first 10 measured frequencies $f_{m}$ for the original frame (undamaged model) are listed in the second column in Table 1. The undamaged frame was numerically analyzed using FE method and its first 10 analyzed frequencies $f_{a}$ are listed in the third column in Table 1. The frequency difference percentages $\Delta f$ and $M A C$ values between the analyzed and measured modes for the original frame are listed in the fourth and fifth column in Table 1, respectively. It can be seen that all of the $\Delta f$ values were low and all of the $M A C$ values were high, hence the FE model simulated the original frame quite well.

The first 10 measured frequencies $f_{m}$ for the damaged frame after cutting are listed in the second column in Table 2 and the corresponding measured mode shapes are shown in Fig. 9. Comparing the measured frequencies between undamaged and damaged models, two features are found: (1) every modal frequency of the frame was decreased after cut; and (2) the frequency changes of the frame were not obvious until about $80 \%$ of strip width was cut. The maximum frequency change was only about $6 \%$.

In order to quantify damage in the cut frame, four 'weak' beam elements were incorporated into the initially undamaged FE model at known locations to simulate the four cuts. The geometrical parameters, cross section area $A_{x}$ and moment of inertia $I_{z}$ of the 'weak' beam elements were selected to update for capturing their 'real' values based on the measured data from the damaged frame. In addition, Young's modulus of steel for all elements was also selected to update globally. The parameter would not be known to 
high accuracy. A small estimation error may account for the small discrepancies between the analytical and experimental results for the undamaged frame. The rest of parameters were all certain and were not updateable; if all parameters were selected for updating locally for every element, than parameters that were certain would end up being updated, with the largest modifications locating the damage. Having located the damage, the certain parameters in the rest of the structure would be reset so that the damage in the identified areas could be quantified. The selected eight geometrical parameters of 'weak' beam elements selected for updating are listed in the second column in Table 3. The measured frequencies and $M A C$ values from the damaged frame were selected as the responses or reference data for the 'damaged' FE model updating. Fig. 6 shows the envelope of normalized relative sensitivities of the responses to the selected eight geometrical parameters; all values of the sensitivity coefficients were rather low. These low values explain why the dynamic properties of the frame changed very little in spite of cutting by about $80 \%$ of the strip width.

Taking reasonable initial values of the updating parameters and doing model tuning based on the measured data from the damaged frame, the selected parameters should be updated close to their real values. The updated frequencies $f_{u}$ are listed in the third column in Table 2. The frequency differences $\Delta f$ and mode shape correlation $M A C$ values between the updated and measured models are listed in the fourth and fifth column in Table 2, respectively. These show maximum frequency difference of only $2 \%$ and very high $M A C$ values larger than $96 \%$. The other ways to correlate the updated data with the measured data are shown in Figures 7 and 8. Fig. 7 shows pairing of frequencies between the updated (FEA) and measured (EMA) models where errors are shown as departures 
from a diagonal line with unit slope. Fig. 8 shows the $M A C$ matrix with high values on its diagonal corresponding to paired modes. The mode shapes of the updated frame model are shown in Fig. 9 and are very close to the measured mode shapes. All of these comparisons between updated and measured data illustrated that the model updating was quite successful.

The updated value of Young's modulus was 194GPa, a reduction of 3\% compared with the initially assumed value $200 G P_{a}$. All selected geometric parameters corresponding to original, damaged and updated are listed in Table 3 . This table provides the database to check the accuracy of damage condition assessment. The second column in Table 3 lists the selected parameters $P$. The third and fourth columns in Table 3 give the values of parameters of the original frame $P_{o}$ and parameters of the damaged frame $P_{c}$, respectively. The parameters' change $\left(P_{o}-P_{c}\right) / P_{o}$ illustrates the damage extent of the frame after cut. The values of updated parameters $P_{u}$ and their differences with the parameters $P_{c}$ of the cut frame $\left(P_{c}-P_{u}\right) / P_{c}$ are listed in the fifth and sixth column in Table 3, respectively. All updated parameters $P_{u}$ values were larger than $P_{c}$ values of the cut frame and both differences were all smaller than $10 \%$. This was due partly to a fact that the width of cut was about $2 \mathrm{~mm}$, whereas the length of 'weak' beam element to represent the cut was $20 \mathrm{~mm}$. Values of the assessed damage quantity $\left(P_{o}-P_{u}\right) / P_{o}$ are listed in the seventh column in Table 3 from which it can be seen that all values were very close to $80 \%$ which was the damage extent of the damaged frame by cut. Judged by these results, the damage assessment for the damaged frame by model updating method provided quantitatively accurate information. 


\section{Limitations to condition assessment by model updating}

Experience in model updating has shown that the limitations tend to be in terms of the ability of the FE model to represent true behaviour, and in the ability to identify a enough modal parameters (frequencies and mode shape ordinates) with sufficient accuracy. The manual tuning includes not just manipulation of structural parameters but also engineering judgment as to the level of detail and reliability of assumptions made in the FE model. For the EMA, even with the best equipment, field conditions may limit the accuracy and resolution (spatially or temporally) of the measurements and statistical uncertainties will carry through to the final structural parameter estimates.

Both aspects, manual tuning, which can be guided by 'expert systems', and reliability of updated parameters are being investigated.

\section{Conclusions}

To implement a successful condition assessment of bridge structures by model updating method, the following specific techniques are of significance:

(1) The FE model for model updating is different from a conventional FE model. In particular the structure should be modelled with as much detail as possible to represent geometric and structural form.

(2) The damaged zones in a structure must be quantified somehow. For this purpose, 'weak' elements can simulate the known or assumed damaged zones very well and updated values of associated parameters represent the damage extents in the damaged structure. 
(3) The selected parameters must be uncertain physical or geometrical properties and should be sensitive to the selected responses. Sensitivity analysis combined with sound engineering judgement is a good way to select updating parameters.

(4) It is necessary to perform limited manual updating based on trial and error to obtain suitable initial values of selected parameters as a starting point for model tuning.

(5) It is possible to identify and quantify damage. 


\section{References}

[1] A.E. Aktan, D.F. Farhey and D.L. Brown, V. Dalal, A.J. Helmicki, V.J. Hunt, S.J. Shelley, "Condition assessment for bridge management", Journal of Infrastructure Systems, ASCE, 2(3), pp. 108-117, 1996.

[2] A.E. Aktan, D.N. Farhey, A.J. Helmicki, D.L. Brown, V.J. Hunt, K.L. Lee and A. Levi, "Structural identification for condition assessment: experimental arts", Journal of Structural Engineering, ASCE, 123(12), pp. 1674-1684, 1997.

[3] A.E. Aktan, N. Catbas, A. Turer and Z. Zhang, "Structural identification: analytical aspects”, Journal of Structural Engineering, ASCE, 124(7), pp. 817-829, 1998.

[4] V.K. Saraf, "Evaluation of existing RC slab bridges", Journal of Performance of Constructed Facilities, ASCE, 12(1), pp. 20-24, 1998.

[5] M.M.A. Wahab and G.D. Roeck, "Damage detection in bridges using modal curvatures: application to a real damage scenario", Journal of Sound and Vibration, 226(2), pp. 217-235, 1999.

[6] J.E. Mottershead and M.I. Friswell, "Model updating in structural dynamics: a survey", Journal of Sound and Vibration, 167(2), pp. 347-375, 1993.

[7] M.I. Friswell and J.E. Mottershead, Finite Element Model Updating in Structural Dynamics, Kluwer Academic Publishers, 1995.

[8] R. Cantieni, "Updating of analytical models of existing large structures based on modal testing", Workshop US-Europe on Bridge Engineering: Evaluation, Management and Repair, ASCE, Barcelona, Spanish, pp. 15-17, 1996. 
[9] A. Pavic, M.J. Hartley and P. Waldron, "Updating of the analytical models of two footbridges based on modal testing of full scale structures", $23^{\text {rd }}$ International Seminar on Modal Analysis, SEM, Leuven, pp. 1111-1118, 1998.

[10] P. Reynolds, A. Pavic and P. Waldron, "Modal testing, FE analysis and FE model correlation of a 600 tonne post-tensioned concrete floor", $23^{\text {rd }}$ International Seminar on Modal Analysis, SEM, Leuven, pp. 1129-1136, 1998.

[11] J.M.W. Brownjohn and P.Q. Xia, "Finite element model updating of a damaged structure", $17^{\text {th }}$ International Modal Analysis Conference, SEM, Kissimmee, Florida, pp. 457-462, 1999.

[12] J.M.W. Brownjohn and P.Q. Xia, "Dynamic assessment of a curved cable-stayed bridge by model updating", Journal of Structural Engineering, ASCE, 126(2), pp. 252-260, 2000.

[13] J.M.W. Brownjohn and P.Q. Xia, "Residual stiffness assessment of failed RC slab by dynamic testing and model updating", submitted to ACI Structural Journal, 2000.

[14] J.M.W. Brownjohn and P.Q. Xia, "Load-carrying capacity assessment of damaged RC beam structure by dynamic testing and model updating", submitted to the Structural Engineer, Journal of the Institution of Structural Engineers, UK, 2000.

[15] R.A. Horn and C.R. Johnson, Matrix Analysis, Cambridge University Press, 1992.

[16] S.S. Rao, The Finite Element Method in Engineering, Butterworth Heinemann, $3^{\text {rd }}$ ed., 1999.

[17] J.M.W. Brownjohn, J. Lee and B. Cheong, "Dynamic performance of a curved cable-stayed bridge”, Engineering Structures, 21(11), pp. 1015-1027, 1999. 
[18] P.Q. Xia, "Condition assessment of bridges by dynamic measurements", Technical Report, School of Civil and Structural Engineering, Nanyang Technological University, Singapore, 1999.

[19] FEMtools user's manual version 1.4, Dynamic Design Solutions, Leuven, Belgium, 1998.

[20] J.M.W. Brownjohn, T.C. Pan and X. Deng, "Correlating dynamic characteristics from field measurements and numerical analysis of a high rise building", Earthquake Engineering and Structural Dynamics, 29(4), pp.523-543, 2000.

[21] R.J. Allemang and D.L. Brown, "A correlation coefficient for modal vector analysis", $1^{\text {st }}$ International Modal Analysis Conference, SEM, Orlando, Florida, pp. 110-116, 1982.

[22] D.J. Ewins, Modal Testing: Theory and Practice, Research Studies Press LTD. John Wiley, 1984. 


\section{List of Notations:}

$$
\begin{array}{ll}
A & =\text { area of cross section } \\
f & =\text { frequency } \\
I & =\text { moment of inertia } \\
M & =\text { number of responses } \\
M A C & =\text { modal assurance criterion } \\
N & =\text { number of parameters } \\
P & =\text { parameter } \\
R & =\text { response } \\
{[S]} & =\text { sensitivity matrix } \\
\Delta & =\text { difference } \\
\phi & =\text { mode shape vector }
\end{array}
$$

\section{Subscripts:}

$\begin{array}{ll}0 & =\text { current iteration } \\ a & =\text { analysis } \\ c & =\text { cut } \\ e & =\text { experiment } \\ i & =i^{\text {th }} \text { response } \\ j & =j^{\text {th }} \text { parameter } \\ m & =\text { measured } \\ n & =\text { normalized } \\ o & =\text { original }\end{array}$




$$
\begin{array}{ll}
r & =\text { relative } \\
u & =\text { updated } \\
x & =\text { coordinator } \\
z & =\text { coordinator }
\end{array}
$$

\section{List of Tables:}

Table 1. Correlation between analyzed and measured modes of undamaged model

Table 2. Correlation between updated and measured modes of damaged model

Table 3. Geometric parameters' values and their changes after updating

\section{List of Figures:}

Fig. 1. A damaged joint model with weak beam

Fig. 2. A damaged bridge FE model with 'weak' beam elements

Fig. 3. FE model of bridge with spine beam

Fig. 4. FE model of bridge with full deck

Fig. 5. Damaged steel portal frame

Fig. 6. Sensitivity envelope of responses to parameters

Fig. 7. Frequency pairing between updated and measured data

Fig. 8. MAC Matrix between updated and measured data

Fig. 9. Mode shapes of damaged portal frame model 
Table 1. Correlation between analyzed and measured modes of undamaged model

\begin{tabular}{|c|c|c|c|c|}
\hline & $\begin{array}{c}\text { Measured } \\
\text { frequencies } \\
f_{m}\end{array}$ & $\begin{array}{c}\text { Analytical } \\
\text { frequencies } \\
f_{a}\end{array}$ & $\Delta f=\frac{f_{a}-f_{m}}{f_{m}}$ & \\
No & $(\mathrm{Hz})$ & $(\mathrm{Hz})$ & $(\%)$ & $\begin{array}{c}M A C \\
(\%)\end{array}$ \\
\hline$(1)$ & $(2)$ & $(3)$ & $(4)$ & $(5)$ \\
\hline 1 & 4.52 & 4.64 & 2.67 & 99.3 \\
\hline 2 & 17.43 & 18.02 & 3.38 & 96.5 \\
\hline 3 & 27.99 & 28.61 & 2.22 & 97.9 \\
\hline 4 & 30.03 & 31.13 & 3.65 & 95.3 \\
\hline 5 & 61.83 & 63.83 & 3.23 & 95.0 \\
\hline 6 & 74.41 & 75.93 & 2.04 & 97.2 \\
\hline 7 & 87.80 & 89.20 & 1.59 & 95.2 \\
\hline 8 & 133.03 & 136.04 & 2.26 & 95.2 \\
\hline 9 & 155.46 & 158.61 & 2.03 & 96.2 \\
\hline 10 & 165.70 & 168.08 & 1.44 & 93.9 \\
\hline
\end{tabular}


Table 2. Correlation between updated and measured modes of damaged model

\begin{tabular}{|c|c|c|c|c|}
\hline No & $\begin{array}{c}\text { Measured } \\
\text { frequencies } \\
f_{m} \\
(\mathrm{~Hz})\end{array}$ & $\begin{array}{c}\text { Updated } \\
\text { frequencies } \\
f_{u} \\
(\mathrm{~Hz})\end{array}$ & $\Delta f=\frac{f_{u}-f_{m}}{f_{m}}$ & $\begin{array}{c}M A C \\
(\%)\end{array}$ \\
\hline (1) & (2) & (3) & (4) & (5) \\
\hline 1 & 4.24 & 4.32 & 1.85 & 99.5 \\
\hline 2 & 16.85 & 16.97 & 0.69 & 99.4 \\
\hline 3 & 26.66 & 26.15 & -1.93 & 96.2 \\
\hline 4 & 29.74 & 29.44 & -1.01 & 96.2 \\
\hline 5 & 60.80 & 61.38 & 0.96 & 98.5 \\
\hline 6 & 71.19 & 70.52 & -0.95 & 97.7 \\
\hline 7 & 85.99 & 85.22 & -0.90 & 97.6 \\
\hline 8 & 130.11 & 130.43 & 0.24 & 97.0 \\
\hline 9 & 152.64 & 152.42 & -0.14 & 96.2 \\
\hline 10 & 162.96 & 161.50 & -0.89 & 96.7 \\
\hline
\end{tabular}


Table 3. Geometric parameters' values and their changes after updating

\begin{tabular}{|c|c|c|c|c|c|c|}
\hline & Parameter & Original & $\operatorname{Cut}\left(\frac{P_{o}-P_{c}}{P_{o}}=80 \%\right)$ & Updated & $\frac{P_{c}-P_{u}}{P_{c}}$ & $\frac{P_{o}-P_{u}}{P_{o}}$ \\
No & $P$ & $P_{o}$ & $P_{c}$ & $P_{u}$ & $(\%)$ & $(\%)$ \\
\hline$(1)$ & $(2)$ & $(3)$ & $(4)$ & $(5)$ & $(6)$ & $(7)$ \\
\hline 1 & $A_{1 x}\left(\mathrm{~m}^{2}\right)$ & $3.0 \times 10^{-4}$ & $6.00 \times 10^{-5}$ & $6.36 \times 10^{-5}$ & -6.00 & 78.80 \\
\hline 2 & $A_{2 x}\left(\mathrm{~m}^{2}\right)$ & $3.0 \times 10^{-4}$ & $6.00 \times 10^{-5}$ & $6.38 \times 10^{-5}$ & -6.33 & 78.73 \\
\hline 3 & $A_{3 x}\left(\mathrm{~m}^{2}\right)$ & $2.4 \times 10^{-4}$ & $4.80 \times 10^{-5}$ & $5.05 \times 10^{-5}$ & -5.21 & 78.96 \\
\hline 4 & $A_{4 x}\left(\mathrm{~m}^{2}\right)$ & $2.4 \times 10^{-4}$ & $4.80 \times 10^{-5}$ & $5.07 \times 10^{-5}$ & -5.62 & 78.88 \\
\hline 5 & $I_{1 z}\left(\mathrm{~m}^{4}\right)$ & $9.0 \times 10^{-10}$ & $1.80 \times 10^{-10}$ & $1.83 \times 10^{-10}$ & -1.67 & 79.67 \\
\hline 6 & $I_{2 z}\left(\mathrm{~m}^{4}\right)$ & $9.0 \times 10^{-10}$ & $1.80 \times 10^{-10}$ & $1.82 \times 10^{-10}$ & -1.11 & 79.78 \\
\hline 7 & $I_{3 z}\left(\mathrm{~m}^{4}\right)$ & $7.2 \times 10^{-10}$ & $1.44 \times 10^{-10}$ & $1.55 \times 10^{-10}$ & -7.64 & 78.48 \\
\hline 8 & $I_{4 z}\left(\mathrm{~m}^{4}\right)$ & $7.2 \times 10^{-10}$ & $1.44 \times 10^{-10}$ & $1.48 \times 10^{-10}$ & -2.78 & 79.44 \\
\hline
\end{tabular}

$P_{o}$ known original value

$P_{c}$ known value after cutting

$P_{u} \quad$ value determined from updating 


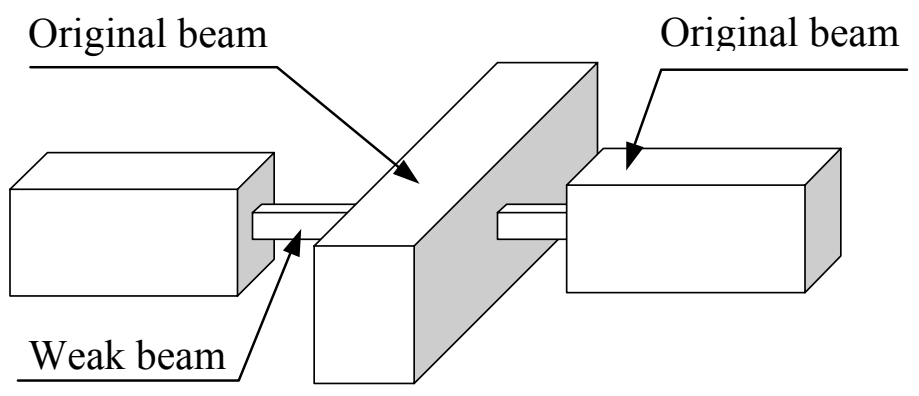

Fig. 1. A damaged joint model with weak beam 


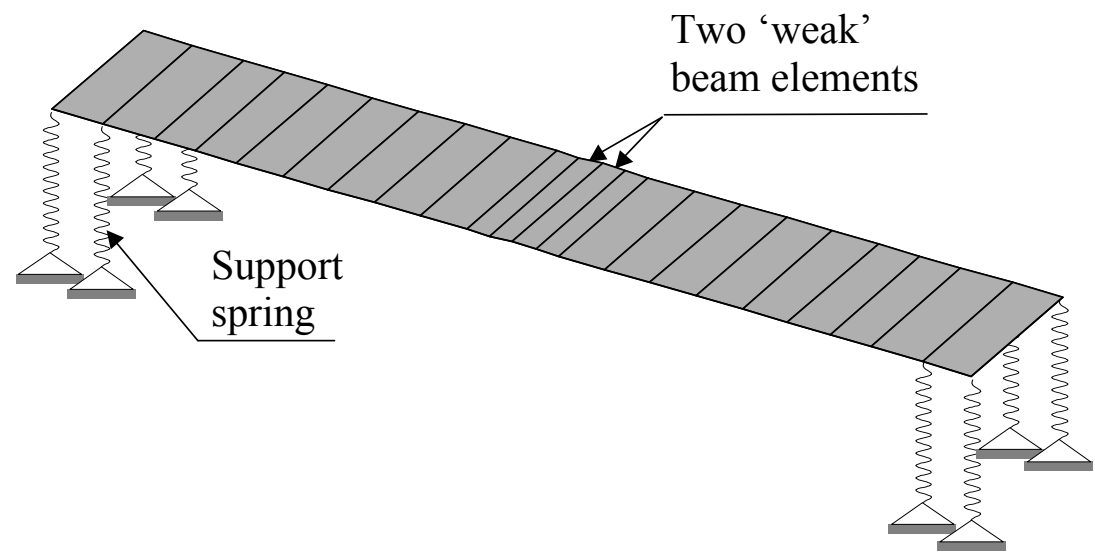

Fig. 2. A damaged bridge FE model with 'weak' beam elements 


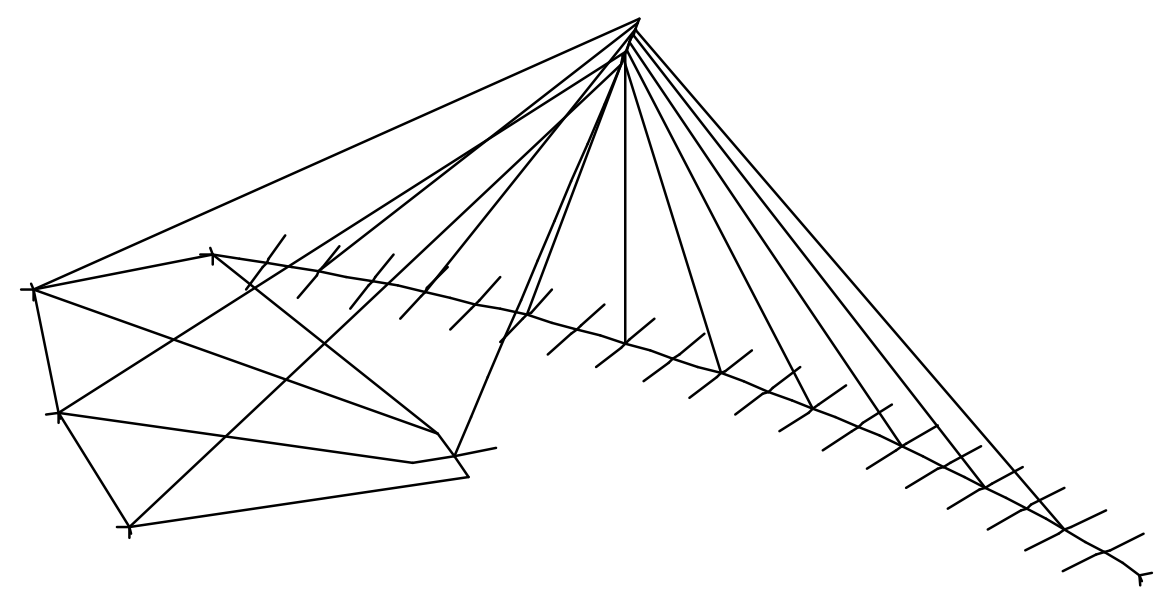

Fig. 3. FE model of bridge with spine beam 


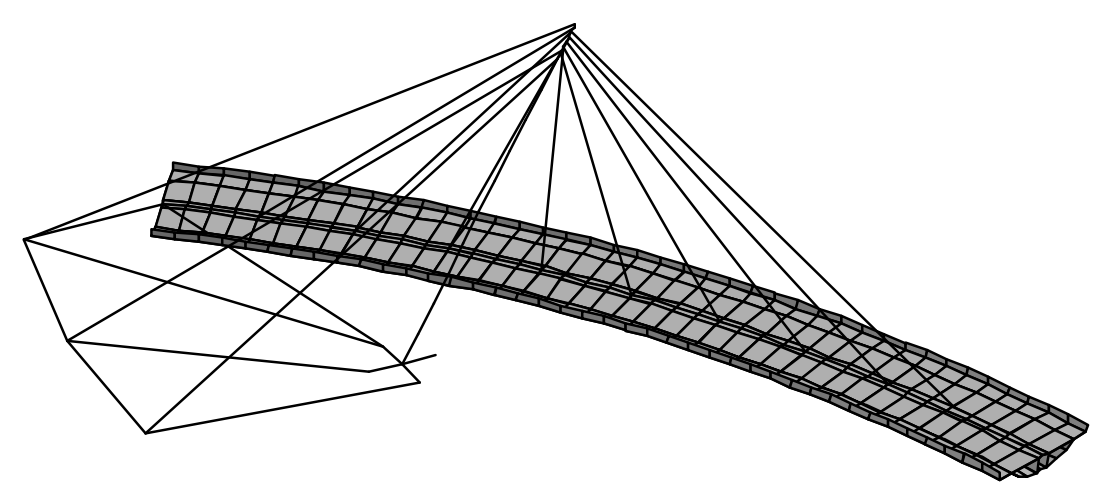

Fig. 4. FE model of bridge with full deck 


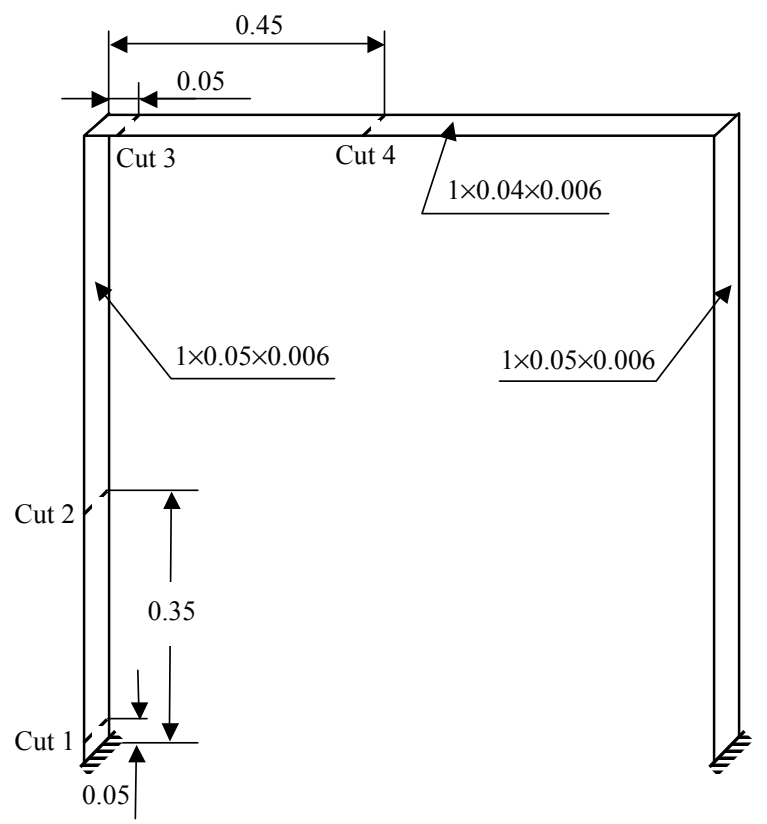

Fig. 5. Damaged steel portal frame (Unit: m) 


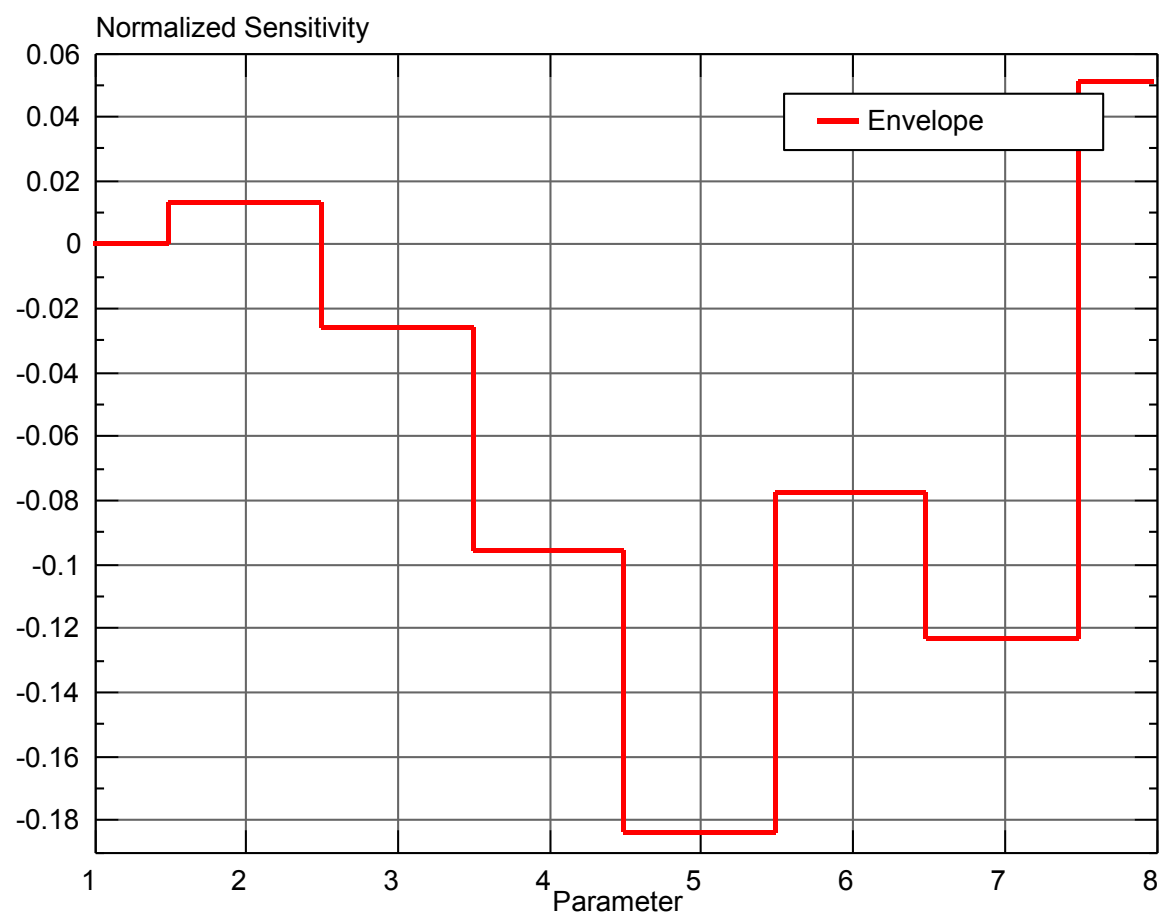

Fig. 6. Sensitivity envelope of responses to parameters 


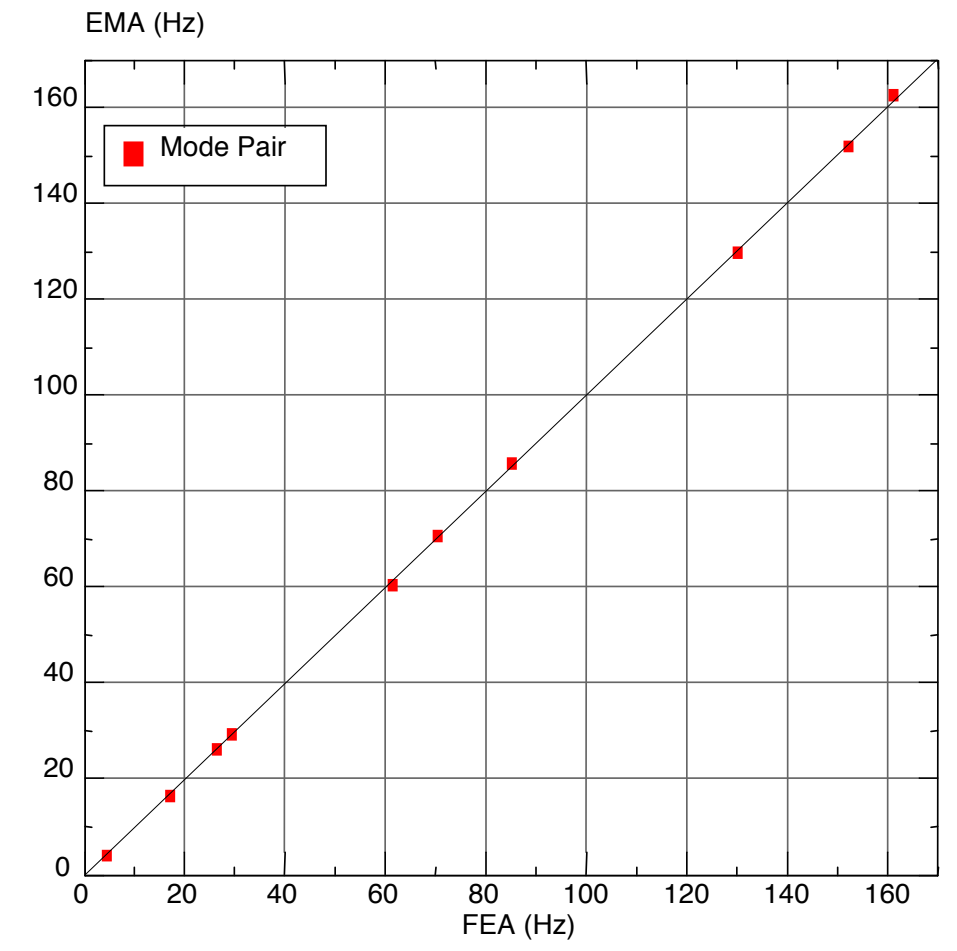

Fig. 7. Frequency pairing between updated and measured data 


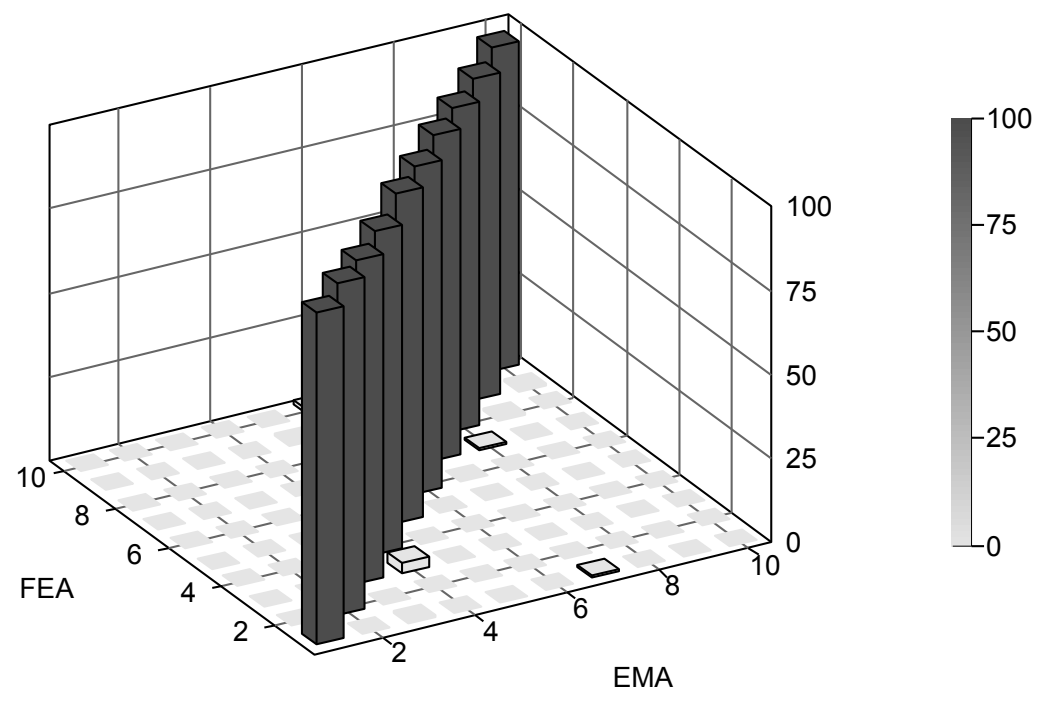

Fig. 8. MAC Matrix between updated and measured data 


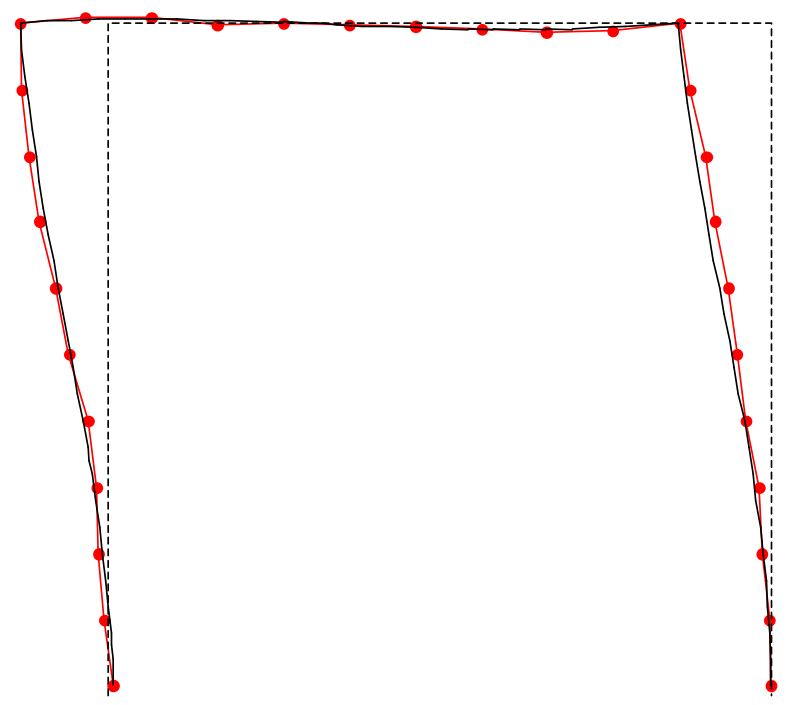

Mode $1(4.24 \mathrm{~Hz})$

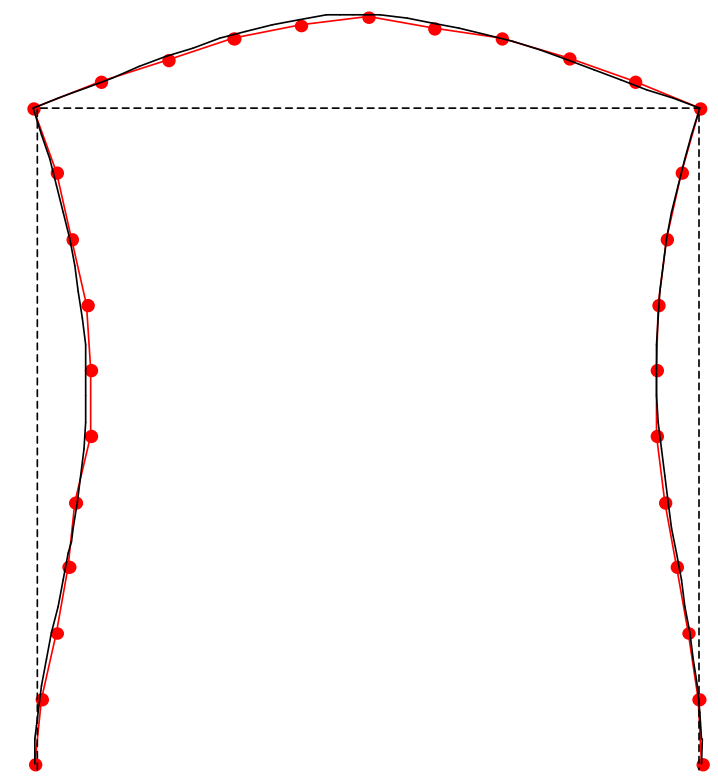

Mode $2(16.85 \mathrm{~Hz})$

Fig. 9. Mode shapes of damaged portal frame model ----undeformed; - updated; $\rightarrow-$ measured 


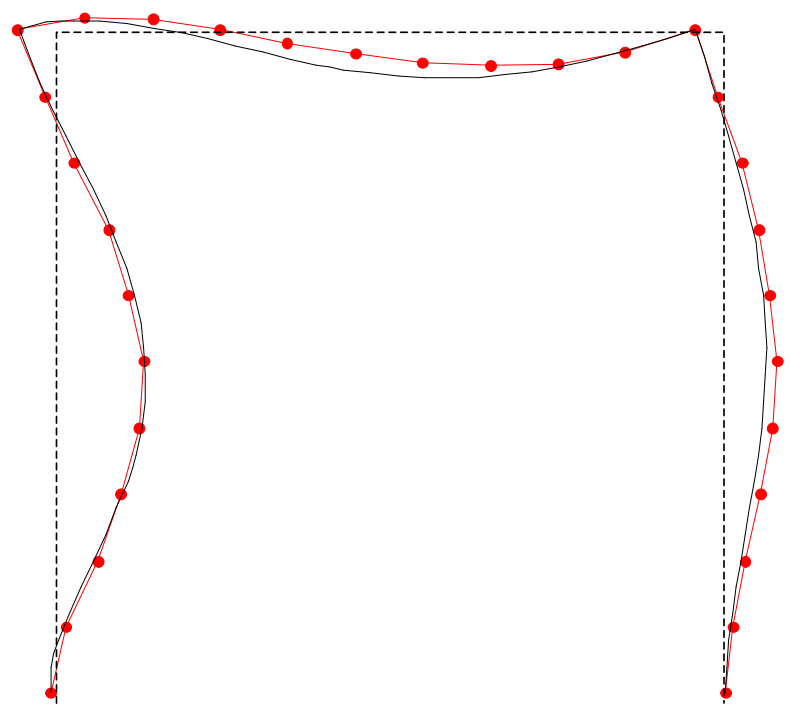

Mode $3(26.66 \mathrm{~Hz})$

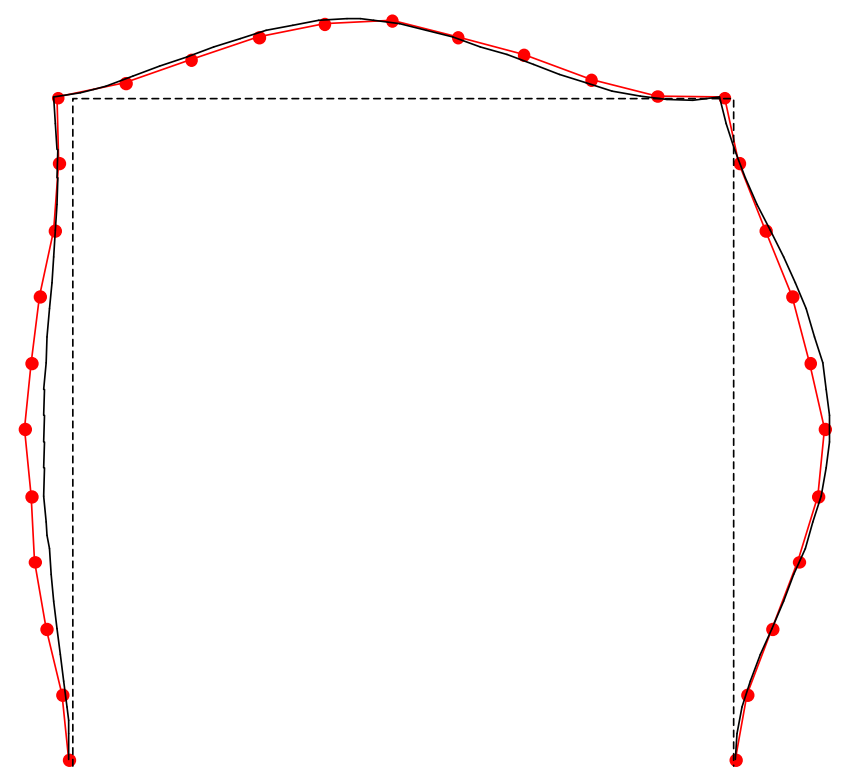

Mode $4(29.74 \mathrm{~Hz})$

Fig. 9. Mode shapes of damaged portal frame model ----undeformed; - - updated; $-\bullet$ measured (Continued) 


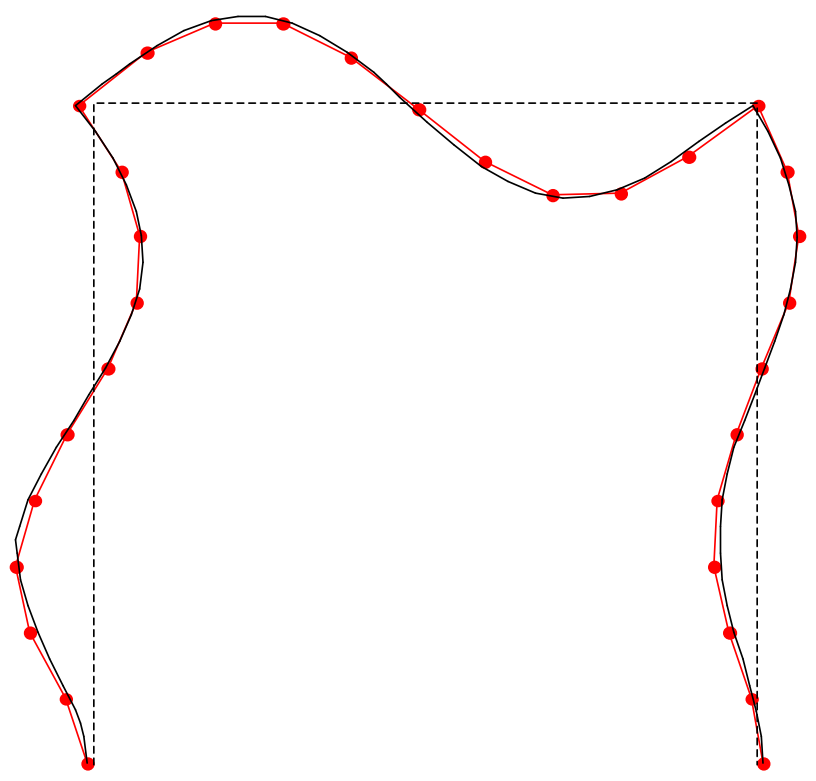

Mode $5(60.80 \mathrm{~Hz})$

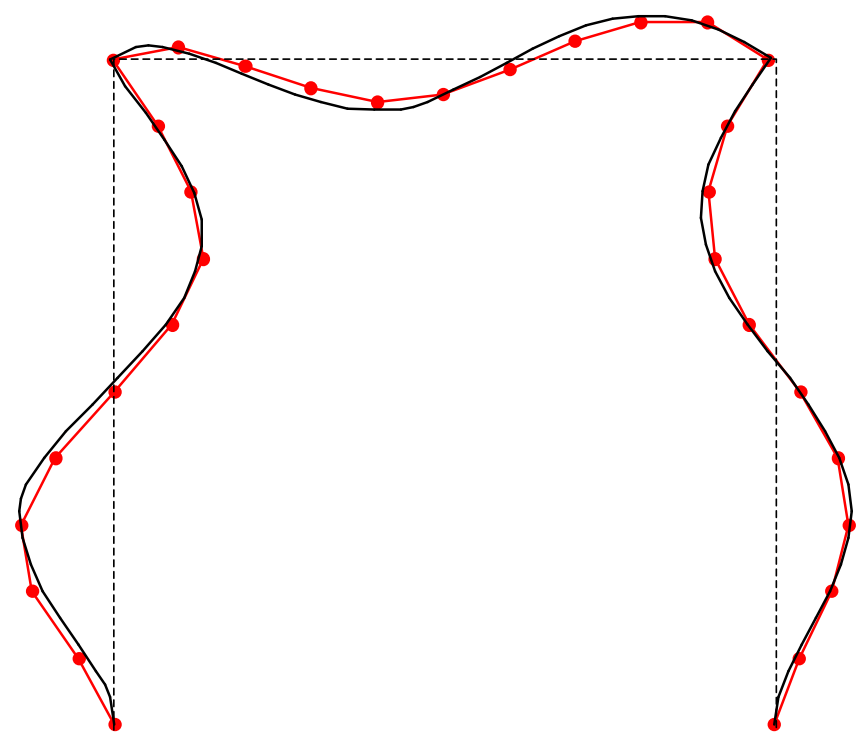

Mode $6(71.19 \mathrm{~Hz})$

Fig. 9. Mode shapes of damaged portal frame model ----undeformed; — updated; $\rightarrow-$ measured (Continued) 


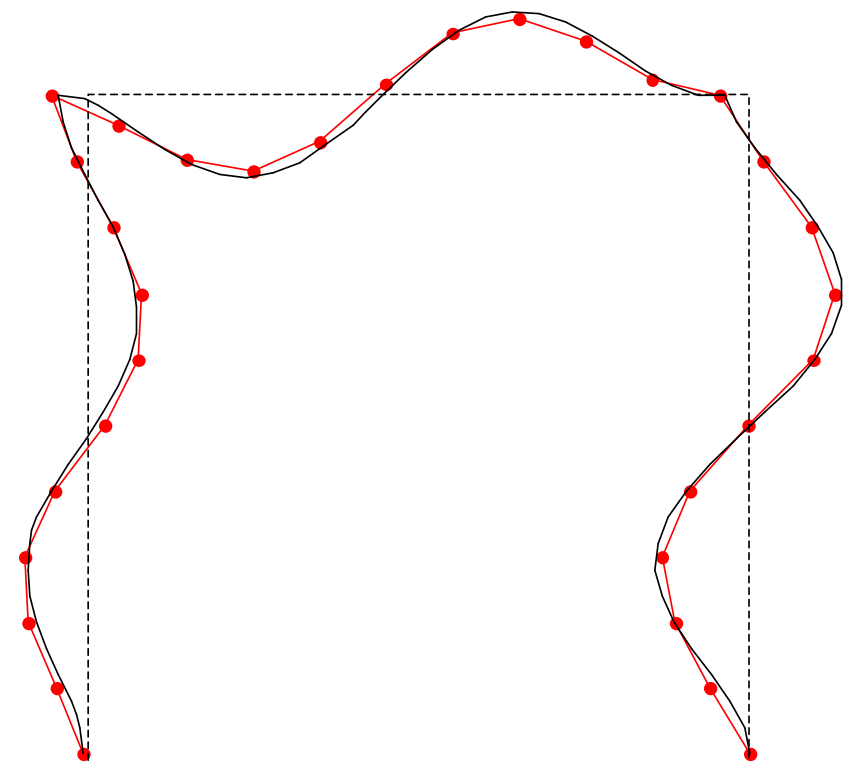

Mode $7(85.99 \mathrm{~Hz})$

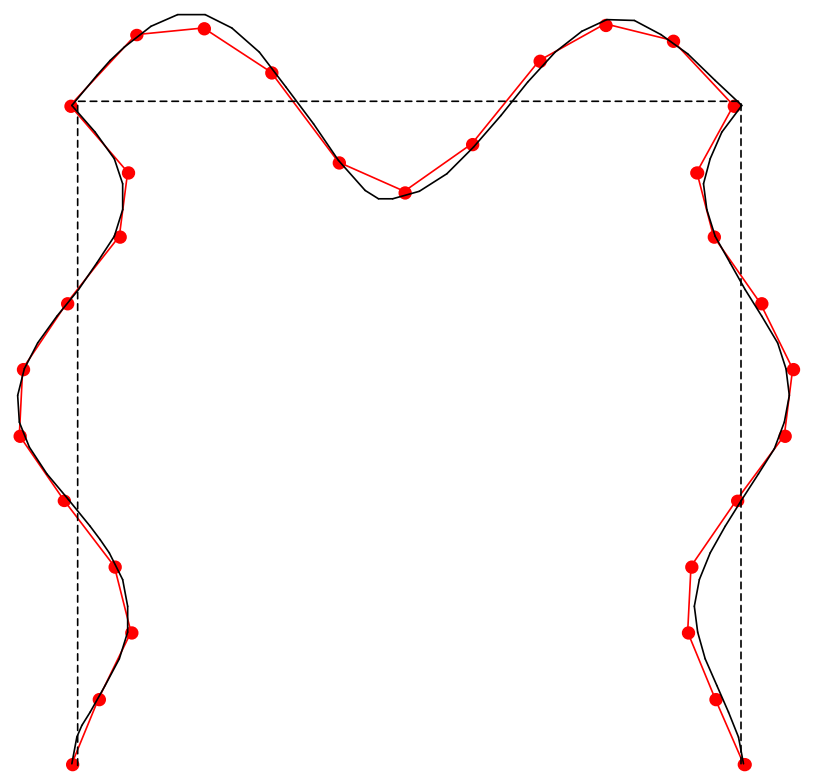

Mode $8(130.11 \mathrm{~Hz})$

Fig. 9. Mode shapes of damaged portal frame model ----undeformed; - updated; $\rightarrow-$ measured (Continued) 


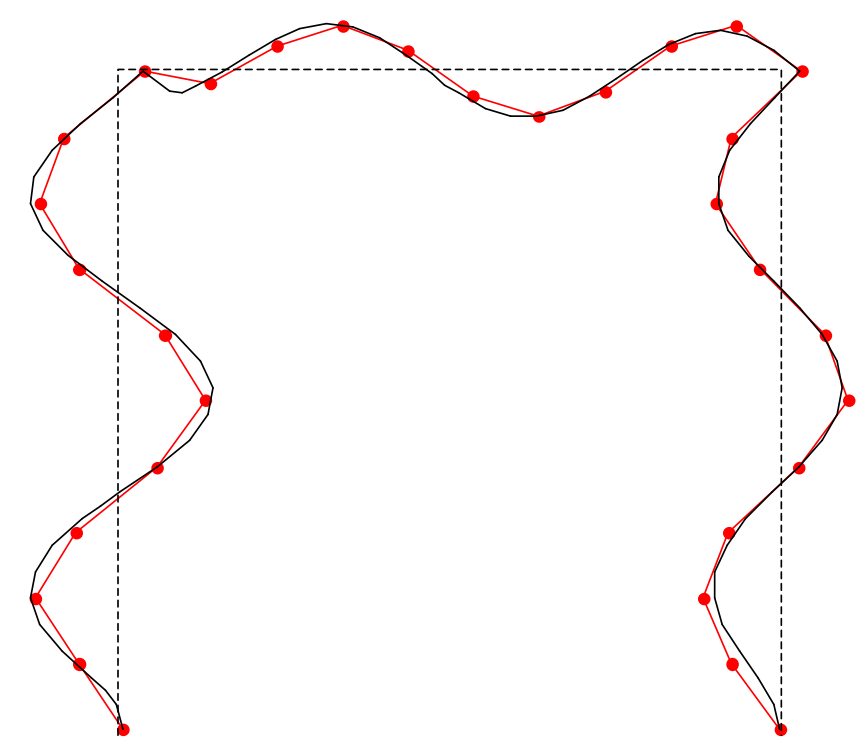

Mode $9(152.64 \mathrm{~Hz})$

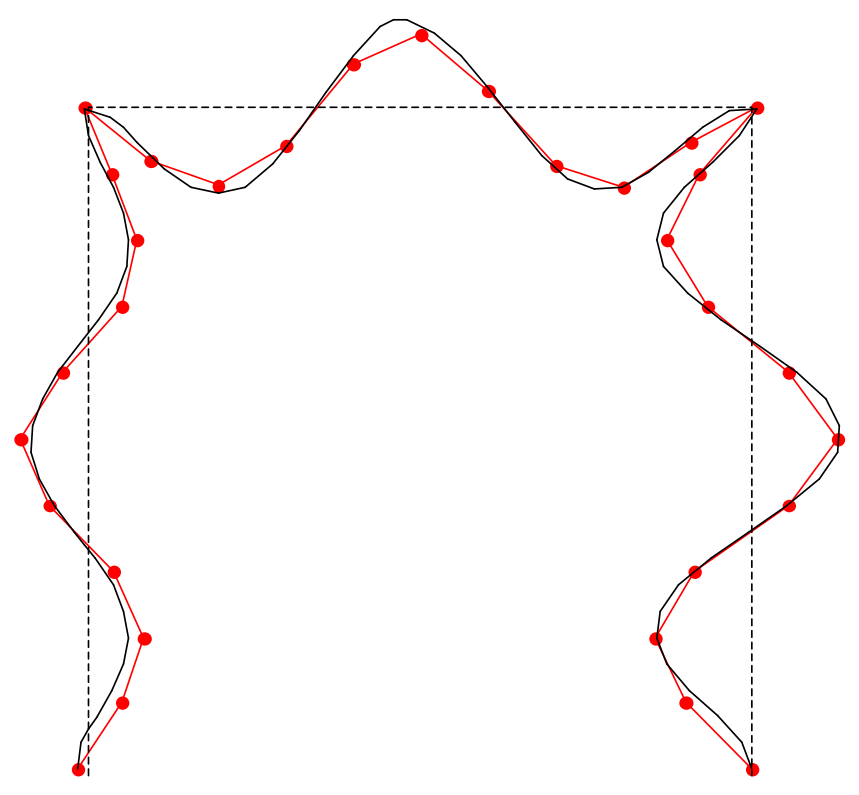

Mode $10(162.96 \mathrm{~Hz})$

Fig. 9. Mode shapes of damaged portal frame model -- undeformed; - updated; - - measured (Continued) 